\title{
Non-Pharmalogical Interventions towards Behavioural and Psychological Symptoms of Dementia-An Integrated Literature Review
}

\author{
Annika Kjallman Alm*, Sandra Danielsson, Lisbeth Porskrog-Kristiansen \\ Department of Nursing, Mid Sweden University, Sundsvall, Sweden \\ Email: *Annika.kjallman-alm@miun.se
}

How to cite this paper: Alm, A.K., Danielsson, S. and Porskrog-Kristiansen, L. (2018) Non-Pharmalogical Interventions towards Behavioural and Psychological Symptoms of Dementia-A Integrated Literature Review. Open Journal of Nursing, 8, 434-447.

https://doi.org/10.4236/ojn.2018.87034

Received: June 15, 2018

Accepted: July 14, 2018

Published: July 17, 2018

Copyright $\odot 2018$ by authors and Scientific Research Publishing Inc. This work is licensed under the Creative Commons Attribution International License (CC BY 4.0).

http://creativecommons.org/licenses/by/4.0/

\begin{abstract}
The objective of this integrated literature review was to identify and examine effectiveness of non-pharmacological interventions towards BPSD. Database searches were documented following the PRISMA flow diagram and included papers were judged by the researchers using the SAHTAASS protocol. Coding of the papers was carried out using Polit and Beck matrix for literature review. It was concluded that several non-pharmacological interventions showed a positive influence on BPSD such as stimulating interventions, social and personalized activities and physical exercise. Also, education/training of healthcare professionals and equipment in form of a structured BPSD protocol resulted in decreased BPSD.
\end{abstract}

\section{Keywords}

BPSD, Complimentary Therapies, Dementia, Integrated Literature Review, Nursing

\section{Introduction}

It is estimated that every 20 year the number of people with dementia diagnosis is about to double. In the year 2050 about 115.4 million people in the world will have some type of dementia [1]. Behavioural and Psychological Symptoms of Dementia (BPSD) are usually the term to describe a group of symptoms that could come with the disease dementia. BPSD includes many different symptoms such as screaming, restlessness, wandering, anxiety, apathy, depression, hallucinations, sleep disturbance and agitation [2] [3]. The frequency and severity of the BPSD symptoms are highly associated with caregiver burden and reducing quality of life for both the person with dementia and their caregivers [2] [4]. 
Medications commonly used for older people with BPSD are of the antipsychotic variety and a chronic use of these drugs is common and is a problem that should be noted [5]. A study demonstrated which side effects antipsychotic drugs can cause people with dementia. Those receiving antipsychotic drugs of the older category, such as Haloperidol, ran between three to four times the risk of dying or ending in hospitals compared to the control group who did not receive any antipsychotic drugs. The most common side effects were hip fractures, bone fracture, stroke or premature death [6].

Antipsychotic drugs should be used with caution after careful consideration between risk and benefit and it is a complex decision involving both the person with dementia, relatives and healthcare professionals. Nurses should be aware of the risks and benefits of antipsychotic drugs as well as alternative non-pharmacological interventions [7]. As the problem has attracted attention, studies around the area have now focused on non-pharmacological interventions. Examples of some of these actions include: music therapy, massage, physical activity, and training and support for staff and family care workers [5]. Studies focusing on alternative methods have shown that several different non-pharmacological interventions can reduce the incidence of BPSD [3].

For health care professionals it is common to meet and nurse people with dementia who are suffering from BPSD. Since there is strong scientific support for interventions towards BPSD to be primarily non-pharmacological, it is important to highlight and summarize the current scientific evidence for these interventions. The objective of this literature review was to identify and examine effectiveness of non-pharmacological interventions towards BPSD.

\section{Method}

\subsection{Selection}

Using an integrated design approach both qualitative and quantitative papers were included, which answered the research question as suggested by Sandelowski et al. [8]. Empirically-based papers of both qualitative and quantitative design were identified through a literature survey in international peer reviewed journals. The specific research question was; which non pharmacological interventions are effective for BPSD? Since the aim of the study also was to highlight and summarize non-pharmacological interventions towards BPSD, a structured integrated literature review was chosen to answer the purpose and highlight current research in the field. The literature searches were conducted in the databases Cinahl, PubMed and PsychInfo. First, broad searches were made, primarily Cinahl-Headings in Cinahl, Mesh terms in PubMed and Thesaurus in PsychInfo. Primary Cinahl-Headings used were: Dementia, behavioral symptoms and alternative therapies. Mesh terms in PubMed: Dementia, behavioral symptoms and complementary therapies. Thesaurus in PsychInfo: Dementia, behavior disorders, intervention, neuropsychiatry and symptoms. In all databases the free text words "BPSD", "neuropsychiatric symptoms" and "non-pharmacological 
interventions" was used. The included papers were published in English and published from January 2012 to February 2017. The process followed the flow outlined in Polit and Beck [9] and were documented by using a Preferred Reporting Items for Systematic Reviews and Meta-Analyses (PRISMA) flow diagram [10] (Please see Figure 1). The included papers had to report from interventional studies, which claimed to address non-pharmacological interventions for BPSD.

\subsection{Validity Assessment}

The included papers were collected and sent to all the research members, who read the included papers and all papers were quality reviewed using the Swedish Agency for Health Technology Assessment and Assessment of Social Services (SAHTAASS) literature review protocol [11]. According to the SAHTAASS [11] all papers were evaluated with the topics: publication year, authors, type of study, purpose, intervention instrument, participants, dropouts, ethical considerations and findings. The studies, which met the inclusion criteria and scored high or medium high quality of the quality assessment, were included in the result.

\subsection{Results of Search Process}

The literature searches were conducted in the databases Cinahl, PubMed and PsychInfo. First, broad searches were made, primarily Cinahl-Headings in Cinahl,

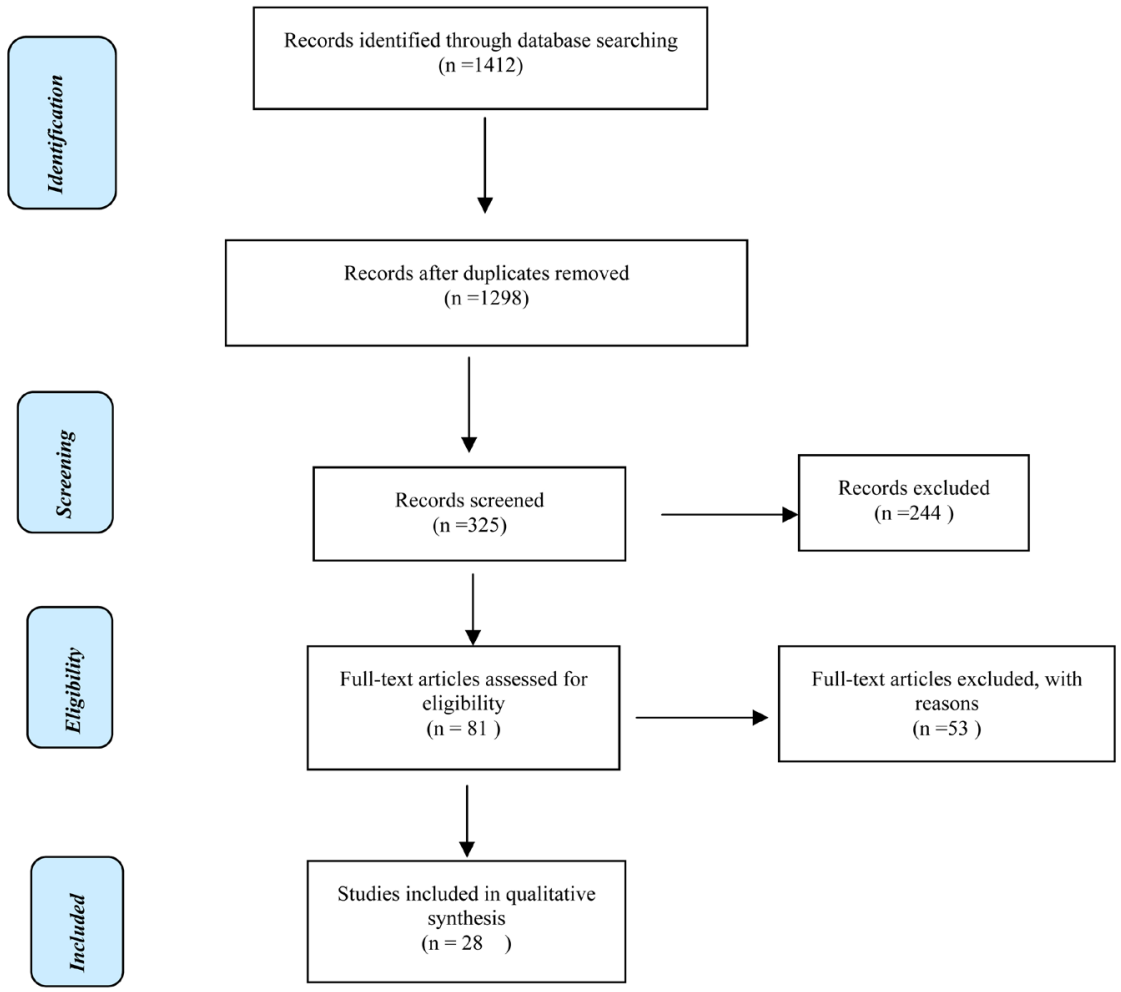

Figure 1. The step taken-using PRISMA Chart flow. 
Mesh terms in PubMed and Thesaurus in PsychInfo. Primary Cinahl-Headings used were: Dementia, behavioral symptoms and alternative therapies. Mesh terms in PubMed were: Dementia, behavioral symptoms and complementary therapies. Thesaurus in PsychInfo: Dementia, behavior disorders, intervention, neuropsychiatry and symptoms. In all databases the free text words "BPSD", "neuropsychiatric symptoms" and "non pharmacological interventions" was used. This was done to get an overview in the area and to study the relevant articles keywords to get additional possible keywords. Then, different combinations of keywords and free text were tested to reduce the risk of missing relevant results. Detailed searches aimed at specific interventions were also conducted based on the results of the broad searches.

The searches were conducted in February and March 2017. Each search in the databases was documented during the work so that the authors could follow which combinations were already made. An example of database search is shown in Table 1.

Table 1. Example of searches in Cinahl.

\begin{tabular}{|c|c|c|c|c|c|c|c|}
\hline Search & Searches Cinahl & $\begin{array}{l}\text { Number of } \\
\text { hits }\end{array}$ & Read titles & Duplicate & Read abstract & $\begin{array}{l}\text { Read articles/quality } \\
\text { examined }\end{array}$ & Included articles \\
\hline N. 1 & \#1 Dementia & 7505 & & & & & \\
\hline N. 2 & $\begin{array}{c}\text { \#1 Dementia AND } \\
\text { \#2Behavioural symptoms } \\
\text { (Heading) }\end{array}$ & 109 & 109 & 0 & 42 & 17 & $\begin{array}{c}\text { Pieper et al. } 2016 \\
\text { Sakamoto et al. } 2013 \\
\text { Van derPloeg et al. } 2012\end{array}$ \\
\hline N. 3 & \#1 AND \#4 BPSD (free text) & 42 & 42 & 3 & 12 & 1 & Raglio et al. 2015 \\
\hline N. 4 & $\begin{array}{c}\text { \#1 AND \#2 AND \#5 } \\
\text { Nonpharmalogiacal } \\
\text { interventions (free text) }\end{array}$ & 5 & 5 & 1 & 3 & 0 & 0 \\
\hline N. 5 & $\begin{array}{l}\text { \#1 AND \#6 Neuropsychiatric } \\
\text { symptoms (free text) }\end{array}$ & 91 & 91 & 3 & 27 & 13 & $\begin{array}{l}\text { Jøranson et al. } 2015 \\
\text { Chen et al. } 2016\end{array}$ \\
\hline N. 6 & $\begin{array}{c}\text { \#1 AND \#3 Alternative } \\
\text { therapies }\end{array}$ & 35 & 35 & 0 & 12 & 6 & Chen et al. 2014 \\
\hline N. 7 & $\begin{array}{l}\text { \#1 AND \#7 Music } \\
\text { Therapy (Heading) }\end{array}$ & 93 & 93 & 7 & 34 & 10 & $\begin{array}{c}\text { Ridder et al. } 2013 \\
\text { Sung et al. } 2012 \\
\text { Wang et al. } 2015 \\
\text { Vink et al. } 2013 \\
\text { Chu et al. } 2014\end{array}$ \\
\hline N. 8 & \#1 AND \#8 Massage (Heading) & 24 & 24 & 3 & 8 & 5 & $\begin{array}{c}\text { Moyle et al. } 2014 \\
\text { Rodriguez-Mansilla et al. } \\
2015 \\
\text { Yang et al. } 2016\end{array}$ \\
\hline N. 9 & $\begin{array}{c}\text { \#1 AND \#9 Phototherapy } \\
\text { (Heading) }\end{array}$ & 6 & 6 & 0 & 4 & 1 & Onega et al. 2016 \\
\hline N. 10 & $\begin{array}{c}\text { \#1 AND \#10 Acupressure } \\
\text { (Heading) }\end{array}$ & 5 & 5 & 1 & 4 & 1 & 0 \\
\hline N. 11 & \#1 AND \#11 Aromatherapy & 17 & 17 & 11 & 7 & 0 & 0 \\
\hline N. 12 & \#1 AND \#12 Staff education & 9 & 9 & 0 & 5 & 2 & 0 \\
\hline N. 13 & \#1 AND \#13 Exercise & 92 & 92 & 0 & 10 & 0 & 0 \\
\hline N. 14 & \#1 AND \#2 AND \#13 & 1 & 1 & 0 & 0 & 0 & 0 \\
\hline N. 15 & \#1 AND \#4 AND \#13 & 70 & 70 & 5 & 4 & 1 & McCabe et al. 2015 \\
\hline
\end{tabular}


At each search, all articles were judged by the title for relevance. At relevant titles, the abstract were read, and the article was subsequently rejected or taken to the next stage of the process. Non-relevant titles and abstracts were excluded.40 papers were excluded due to poor quality either in design of intervention or intervention instrument. 28 papers met the inclusion criteria as described in the PRISMA flow chart in Figure 1.

\subsection{Data Analysis}

Using a coding process as described by Polit and Beck ([9], pp. 98-99), the authors systematically classified data, extracted and identified patterns within the findings, and discussed categories emerging from the articles findings. 28 scientific articles resulted in four categories and five subcategories: Sensory stimulating interventions with subcategories; music therapy in-group, individual music therapy, massage and acupressure, aromatherapy, light therapy, social and individual interventions, physical training and education for healthcare professionals.

\section{Results}

\subsection{Sensory Stimulating Interventions}

\subsubsection{Music Therapy In-Group}

Musical therapy in-group has been shown to reduce the symptoms of anxiety within BPSD [12] [13]. Group therapy has also been shown to result in a significant decrease in agitation, as well as an improvement in the overall mood of people with dementia and reduced the severity of behavioral disorders [14] [15]. Sung et al. [12], however, did not found that music therapy gave any significant difference regarding the symptom of agitation. Chu et al. [16] states that music therapy for elderly people with dementia has been shown to significantly reduce depression. Music activities that were given regularly had a relaxing effect and generally affected their mood to the positive side and reduced the level of depressive symptoms. However, in the study made by Wang et al. [13] symptoms of depression between intervention group and control group showed no significant differences. On the other hand, participating in music therapy gave significant differences in the scale of behavioral disorders, CAPE-BRS (Clifton Assessment Procedures for the Elderly Behavior Rating Scale).

\subsubsection{Individual Music Therapy}

Music interventions with customizable music that recreate memories can reduce stress and increase relaxation in people with severe dementia. Not only listening to individualized music but also activating it such as singing and dancing to the music gave a significant decrease in BPSD. However, three weeks after intervention, the effect disappeared [17]. Individual music therapy has been shown to reduce agitation in people with dementia as compared to a control group. The effect of reducing agitation was that prescription medication lessened among the people involved in the music intervention [18]. Healthcare professionals have 
also reported on perceived improvements after music therapy, including agitation, anxiety and apathy in people with dementia [19]. Researchers have also compared the effects of a multi-sensory stimulating environment to individualized music sessions. The result showed that the multisensory environment had a better effect on anxiety than the music sessions. As for agitation, both groups had improved results [20]. However, Raglio et al. [21] showed a different result. Their study was based on random participants being divided into three groups: one who received regular care plus active music therapy, one who received regular care plus they listened to individually customized music (alone) and a group who received only normal care without any music. The result showed that all three groups had significant improvements in BPSD.

\subsubsection{Massage and Acupressure}

Acupressure and relaxing massage has been shown to be more effective in improving depression and anxiety in people with dementia than those who have not received any of these treatments. However, acupressure gave greater effect to the symptoms than massage, indicating that acupressure is a more effective intervention [22]. Acupressure combined with lavender oil has shown significant results in reducing agitation, agitation decreased after first treatment and lasted three weeks after intervention [23]. In another study, the researchers tested the comparison of foot massage with a person sitting alone with the person in a room without speaking (quiet presence) of agitation in people with dementia. The result showed that agitation increased in both groups but that the increase was greater in the group who did not receive foot massage. Foot massage, however, gave no significant decrease in agitation of the person with dementia disease [24].

\subsubsection{Aromatherapy}

Placing lavender oil on the skin, as compared to regular oil (placebo) has not been shown to improve or reducing the frequency of agitated behavior in people with dementia [25]. Aromatherapy with lavender oil in another study, however, showed improvements in agitation, the treatment made people with dementia more relaxed and that reduced the symptom. However, aromatherapy in combination with acupressure had an even greater effect [23]. Aromatherapy in combination with massage has been shown to give a significant difference in depressive symptoms in comparison to the standardized control group. Regarding the symptom of agitation, aromatherapy combined with massage overall did not improve over a lengthy period, however, differences were detected within 24 hours after intervention during certain weeks of the intervention. The conclusion was that aromatherapy in combination with massage can be an effective and safe intervention for specific agitated behaviors and depression in individuals with dementia [23]. Another study by Fu, Moyle and Cooke [26] examined whether aromatherapy and hand massage could reduce agitated behaviors in people with dementia. Despite a downward trend in agitating behavior, re- 
searchers could not demonstrate that there was a significant decrease in any of the groups.

\subsubsection{Light Therapy}

Onega, Pierce and Epperly [27] examined the effects of light therapy on agitation and depression in people with dementia. The result showed that exposure of light resulted in significant improvements in both agitation and depression while those receiving placebo showed elevated levels of symptoms or no difference before and after.

\subsection{Social and Individual Interventions}

Both individually adapted activities and other general activities that include social contact showed reduced agitation in people with dementia disease [28] [29] [30]. Activities based on people's past interests and hobbies gave improvement in agitation in individuals who did not speak English or had lost the ability to speak [28]. An activity program containing music, orientation training, physical activity and art-based cognitive exercise, significantly improved BPSD in men with dementia compared with the control group. The researchers concluded that these non-pharmacological interventions had a positive effect on BPSD [28]. Activity in a group with a robotic animal showed three months after intervention a significant reduction in the symptoms of agitation and depression in people with dementia [29]. Also, a study by Vink et al. [15] showed that group activities such as cooking, handwork and puzzles gave a short-lived reduction in agitation in people with dementia. Treush et al. [31] showed that apathy in the control group was significantly greater than the group who received individually tailored activities. The intervention was effective when implemented, but the result was not lasting one year afterwards. In another study by Chen, Tsai, Chao and Clark [32], the researchers used a person-centered approach in which people with dementia, received therapy that focused on learning. The result showed that the therapy significantly improved symptoms such as hallucinations, bizarre behavior, depression, apathy, irritation and sleep problems.

\subsection{Physical Training}

Physical exercise with medium intensity for 60 minutes three times a week in people with Alzheimer's disease resulted in a significant decrease in the Neuropsychiatric Inventory (NPI) scale for BPSD. However, it did not affect depressive symptoms [33]. Another study examined the effects of a high intensity exercise program on depressive symptoms in people with dementia. The participants in the experimental group exercised every other day for four months while the control group had other activities such as conversation and reading. The result showed no significant difference between the training group and the control group. However, participants with highly estimated depressive symptoms initially had significant differences at the end of the study in both groups, which may mean that both physical activity and non-physical activities can reduce 
highly estimated depressive symptoms [34]. Chen et al. (2017) [35] conducted a study where people with dementia who were sitting in a wheelchair trained with resistance bands. The study showed that the intervention gave significant results regarding decreased depressive symptoms as well as decreased behavioral problems compared to the control group. Lowery et al. [36] examined the effects of lighter training in the form of walking as therapy for BPSD but the result did not show any significant differences on the NPI scale.

\subsection{Education/Training for Healthcare Professionals}

Training for healthcare professionals in dealing with BPSD leads to improvements in the symptoms of people with dementia disease [37] [38]. When healthcare professionals received training, exercise and equipment in the form of a structured BPSD protocol the result was improvements in behavioral symptoms in people with dementia. Personnel who also received clinical support from a BPSD expert achieved better results than those who did not receive support [37]. Training the staff in managing BPSD also resulted in the use of psychotropic drugs to a lesser extent [38].

\section{Discussion}

The aim of the review was to identify and examine effectiveness of non-pharmacol ogical interventions towards BPSD. The result showed that non-pharmacological interventions stimulated the senses such as hearing, feeling and sight can alleviate and reduce BPSD in people with dementia. Music therapy, massage/acupressure and aromatherapy suggest being good interventions while some studies have not been able to show significant results for this. Vink, Zuidersma, Zuidema and Slaets [15] agree that music therapy is a powerful intervention to reduce BPSD. In their study, participants who received music therapy had a significant improvement on the NPI scale compared to the control group.

Only one study in the result describes the effects of light therapy and that support the use of light to reduce depression and agitation. Figuero et al. [39] described how the participants through light therapy received increased night sleep and reduced symptoms of agitation and depression. However, a previous study by Hickman et al. [40] found no support for light therapy to affect depressive symptoms in people with dementia. Perhaps in the seven years between the studies the effectiveness of light therapy improved since 2007 could be one explanation to this.

In the result, it appears that aromatherapy had a positive effect in one of three studies. Because of that, the effect of the intervention on BPSD feels doubtful. In another study by Yoshiyama, Arita and Suzuki [41], researchers were also unable to provide evidence that aromatherapy gave significant improvements in BPSD.

The result showed that both individualized activities and other general activities that include social contact could reduce the incidence of BPSD. Van Haitsma et al. [42] also demonstrated the value of individualized activities and how in- 
terventions for individuals affected with dementia can increase well-being and reduce aggressiveness.

Physical exercise has been shown to decrease BPSD, but it has also been shown to not make any significant difference. In a literature study by Thuné-Boyle et al. [43], the authors stated that physical exercise might reduce BPSD, especially symptoms such as sedation, agitation, walking behavior and sleeping problems. However, for other symptoms such as anxiety, apathy and decreasing repetitive behavior, the evidence was low.

The results support that training and education for healthcare professionals in managing BPSD leads to improvements in the symptoms of people with dementia. Kristiansen et al. [4] consider that one possible way to reduce the prevalence of BPSD-related violence is to develop education, leadership, supervision and reflection. This could also be a way to increase the well-being of the nurses and increase their job satisfaction.

The authors of this literature review consider that the health care professional's influence on the person with BPSD should not be neglected. With education, supervision and possibility to practice under guidance of understanding and with the right tools (for example a structured BPSD protocol), the health care staff can do a significant difference for the person with dementia. In order to make a sustainable change in approach and understanding, the authors believe that the health care providers need to obtain embodied experience of meeting people with BPSD. This demands that academic facilities and health care employers with join forces take on this challenge and educational responsibility. The Swedish Society of Nursing [44] connects the nursing profession ability to provide individualized person-centred care with the ability to deliver a safe health care and as a strategy for improving the quality of nursing. One way to get understanding for the individual is by taking part of the individual's life story. The nurse can do this by interviewing the person with dementia and his/her relatives. A study made by Novy [45] suggests that life story fosters opportunities for meaningful communication among and between people living with dementia. Kitwood ([46], p. 105) also writes that healthcare professionals should work for an environment where every person with dementia are seen as a unique individual and that this can be achieved by the staff through a combination of empathy and knowledge.

The result of the present review suggests that non-pharmacological interventions at BPSD should be based on a person-centred care. Kolanowski, Fick, Frazer and Penrod [47] support non-pharmacological interventions at BPSD and the importance of staff knowing the individual so that interventions can be personalized according to the person's background and interests.

\section{Limitations}

Since the aim of the study was to highlight and summarize effectiveness of non-pharmacological interventions towards BPSD, an integrated literature re- 
view was preferred because it aims to give the reader an objective and organized synthesis of evidence within the subject.

The data collection was preformed in the databases Cinahl, PubMed and PsychInfo. Keywords in each database gave relevant hits with articles that corresponded to the purpose of the study. The definitions of years and languages were made because the authors wanted the study to be based on the latest research. No delimitation was made related to countries because the authors wanted a global perspective. Included articles in the results came from a total of 13 different countries.

The articles were quality examined using SAHTAASS: literature review protocol [11]. The protocol are used to support structured assessment of the quality of the articles, and although the authors have tried to review all articles objectively, the assessment will always be subjective, which could be a weakness. A review protocol that gave a total amount of points regarding the quality of the article might have facilitated the review. Seven of the articles were classified as high quality and 21 as medium high. The large number of medium high articles could be considered as a weakness in the review.

\section{Conclusion}

Several non-pharmacological interventions were found to have a positive impact on BPSD, but interventions should be adapted to the individual and it is important that the nurses in care of people with dementia and healthcare staff is working by a person-centred care. This is to find the right interventions for the right individual to get the desired effect. This result strengthens and goes in line with previous research that shows that person-centred nursing has the potential to reduce BPSD [47] [48].

\section{Acknowledgements}

We would like to thank Region Jämtland and Mid Sweden University, Department of Nursing science, for supporting this academic work.

\section{References}

[1] Prince, M., Bryce, R., Albanese, E., Wimo, A., Ribeori, W. and Ferri, C.P. (2013) The Global Prevalence of Dementia: A Systematic Review and Metaanalysis. Alzheimer's \& Dementia, 9, 63-75. https://doi.org/10.1016/j.jalz.2012.11.007

[2] Finkel, S. (2000) Introduction to Behavioural and Psychological Symptoms of Dementia (BPSD). International Journal of Geriatric Psychiatry, 15, S2-S4.

[3] Martini de Oliveira, A., Radanovic, M., Homem de Mello, P.C., Cardoso Buchain, P., Dias Barbosa Vizzotto, A., Calestino, D.L., Stella, F., Piersol, C.V. and Forlenza, O.V. (2015) Nonpharmacological Interventions to Reduce Behavioral and Psychological Symptoms of Dementia: A Systematic Review. BioMed Research International, 2015, 1-9. https://doi.org/10.1155/2015/218980

[4] Kristiansen, L., Hellzén, O. and Asplund, K. (2006) Swedish Assistant Nurses' Experiences of Job Satisfaction When Caring for Persons Suffering from Dementia and Behavioural Disturbances, International Journal of Qualitative Studies on Health 
and Well-Being, 1, 245-256. https://doi.org/10.1080/17482620600601187

[5] Azermai, M. (2015) Dealing with Behavioral and Psychological Symptoms of Dementia: A General Overview. Psychology Research and Behavior Management, 8, 181-185. https://doi.org/10.2147/PRBM.S44775

[6] Rochon, P.A., Normand, S.-L., Gomes, T., Gill, S.S., Anderson, G.M., Melo, M., Sykora, K., Lipscombe, L., Bell, C.M. and Gurwitz, J.H. (2008) Antipsychotic Therapy and Short-Term Serious Events in Older Adults with Dementia. Archives of Internal Medicine, 168, 1090-1096. https://doi.org/10.1001/archinte.168.10.1090

[7] Howland, R.H. (2008) Risks and Benefits of Antipsychotic Drugs in Elderly Patients with Dementia. Journal of Psychosocial Nursing and Mental Health Services, 46, 19-23.

[8] Sandelowski, M., Voils, C.I. and Barroso, J. (2006) Defining and Designing Mixed Research Synthesis Studies. Research in the Schools: A Nationally Refereed Journal Sponsored by the Mid-South Educational Research Association and the University of Alabama, 13, 29.

[9] Polit, D.F. and Tatano Beck, C. (2017) Nursing Research. Generating and Assessing Evidence for Nursing Practice. Wolters Kluwer Health, Philadelphia.

[10] Moher, D., Liberati, A., Tetzlaff, J., Altman, D.G. and The PRISMA Group (2009) Preferred Reporting Items for Systematic Reviews and Meta-Analyses: The PRISMA Statement. PLoS Medicine, 6, e1000097. https://doi.org/10.1371/journal.pmed.1000097

[11] SBU (2014) Utvärdering av metoder i hälso-och sjukvården: En handbok. 2 uppl. Stockholm: Statens beredning för medicinsk utvärdering (SBU). http://www.sbu.se/globalassets/ebm/metodbok/sbushandbok.pdf

[12] Sung, H.-C., Lee, W.-L., Li, T.-L. and Watson, R. (2012) A Group Music Intervention Using Percussion Instruments with Familiar Music to Reduce Anxiety and Agitation of Institutionalized Adults with Dementia. International Journal of Geriatric Psychiatry, 27, 621-627. https://doi.org/10.1002/gps.2761

[13] Wang, S.-C., Yu, C.-L. and Chang, S.-H. (2015) Effect of Music Care on Depression and Behavioral Problems in Elderly People with Dementia in Taiwan: A Quasi-Experimental, Longitudinal Study. Aging \& Mental Health, 21, 156-162. https://doi.org/10.1080/13607863.2015.1093602

[14] Narme, P., Clément, S., Ehrlé, N., Schiaratura, L., Vachez, S., Courtaigne, B., Munsch, F. and Samson, S. (2014) Efficacy of Musical Interventions in Dementia: Evidence from a Randomized Controlled Trial. Journal of Alzheimer's Disease, 38, 359-369. https://doi.org/10.3233/JAD-130893

[15] Vink, A.C., Zuidersma, M., Boersma, F., de Jonge, P., Zuidema, S.U. and Slaets, J.P. J. (2013) The Effect of Music Therapy Compared with General Recreational Activities in Reducing Agitation in People with Dementia: A Randomised Controlled Trial. International Journal of Geriatric Psychiatry, 28, 1031-1038. https://doi.org/10.1002/gps.3924

[16] Chu, H.C., Yang, C.-Y., Lin, Y., Ou, K.-L., Lee, T.-Y., O’Brien, A.P. and Chou, K.-R. (2014) The Impact of Group Music Therapy on Depression and Cognition in Elderly Persons with Dementia: A Randomized Controlled Study. Biological Research for Nursing, 16, 209-217. https://doi.org/10.1177/1099800413485410

[17] Sakamoto, M., Ando, H. and Tsutou, A. (2013) Comparing the Effects of Different Individualized Music Interventions for Elderly Individuals with Severe Dementia. International Psychogeriatrics, 25, 775-784. https://doi.org/10.1017/S1041610212002256 
[18] Ridder, H.M.O., Stige, B., Qvale, B.S. and Gold, C. (2013) Individual Music Therapy for Agitation in Dementia: An Exploratory Randomized Controlled Trial. Aging \& Mental Health, 17, 667-678. https://doi.org/10.1080/13607863.2013.790926

[19] Hsu, M.H., Flowerdew, R., Parker, M., Fachner, J. and Odell-Miller, H. (2015) Individual Music Therapy for Managing Neuropsychiatric Symptoms for People with Dementia and Their Careers: A Cluster Randomised Controlled Feasibility Study. BMC Geriatrics, 15, 1-19.

[20] Sánchez, A., Maseda, A., Marante-Moar, M.P., de Labra, C., Lorenzo-López, L. and Millán-Calenti, J.C. (2016) Comparing the Effects of Multisensory Stimulation and Individualized Music Sessions on Elderly People with Severe Dementia: A Randomized Controlled Trial. Journal of Alzheimer's Disease, 52, 303-315. https://doi.org/10.3233/JAD-151150

[21] Raglio, A., Bellandi, D., Baiardi, P., Gianotti, M., Ubezio, M.C., Zanacchi, E., Granieri, E., Imbriani, M. and Stramba-Badiale, M. (2015) Effect of Active Music Therapy and Individualized Listening to Music on Dementia: A Multicenter Randomized Controlled Trial. Journal of the American Geriatrics Society, 63, 1534-1539. https://doi.org/10.1111/jgs.13558

[22] Rodríguez-Mansilla, J., López-Arza, M.V.G., Varela-Donoso, E., Montanero-Fernández, J., Sánchez, B.G. and Garrido-Ardila, E.M. (2015) The Effects of Ear Acupressure, Massage Therapy and No Therapy on Symptoms of Dementia: A Randomized Controlled Trial. Clinical Rehabilitation, 29, 683-693.

https://doi.org/10.1177/0269215514554240

[23] Yang, M.-H., Lin, L.-C., Wu, S.-C., Chiu, J.-H., Wang, P.-N. and Lin, J.-G. (2015) Comparison of the Efficacy of Aroma-Acupressure and Aromatherapy for the Treatment of Dementia-Associated Agitation. BMC Complementary \& Alternative Medicine, 15, 93.

[24] Moyle, W., Cooke, M.L., Beattie, E., Shum, D.H.K., O’Dwyer, S.T. and Barrett, S. (2013) Foot Massage versus Quiet Presence on Agitation and Mood in People with Dementia: A Randomised Controlled Trial. International Journal of Nursing Studies, 51, 856-864. https://doi.org/10.1016/j.ijnurstu.2013.10.019

[25] O’Connor, D.W., Eppingstall, B., Taffe, J. and van derPloeg, E.S. (2013) A Randomized, Controlled, Cross-Over Trial of Dermally-Applied Lavender (Lavandula angustifolia) Oil as a Treatment of Agitated Behaviour in Dementia. BMC Complementary \& Alternative Medicine, 13, 315.

[26] Fu, C.-Y., Moyle, W. and Cooke, M. (2013) A Randomised Controlled Trial of the Use of Aromatherapy and Hand Massage to Reduce Disruptive Behaviour in People with Dementia. BMC Complementary and Alternative Medicine, 13, 165. https://doi.org/10.1186/1472-6882-13-165

[27] Onega, L.L., Pierce, T.W. and Epperly, L. (2016) Effect of Bright Light Exposure on Depression and Agitation in Older Adults with Dementia. Issues in Mental Health Nursing, 37, 660-667. https://doi.org/10.1080/01612840.2016.1183736

[28] Chen, R.-C., Liu, C.-L., Lin, M.-H., Peng, L.-N., Chen, L.-Y., Liu, L.-K. and Chen, L.-K. (2014) Non-Pharmacological Treatment Reducing Not Only Behavioral Symptoms, But Also Psychotic Symptoms of Older Adults with Dementia: A Prospective Cohort Study in Taiwan. Geriatrics Gerontology International, 14, 440-446. https://doi.org/10.1111/ggi.12126

[29] Jøranson, N., Pedersen, I., MorkRokstad, A.-M. and Ihlebæk, C. (2015) Effects on Symptoms of Agitation and Depression in Persons with Dementia Participating in Robot-Assisted Activity: A Cluster-Randomized Controlled Trial. Journal of the 
American Medical Directors Association, 16, 867-873.

[30] Van der Ploeg, E.S., Eppingstall, B., Camp, C.J., Runci, S.J., Taffe, J. and O’Connor, D.W. (2013) A Randomized Crossover Trial to Study the Effect of Personalized, One-to-One Interaction Using Montessori-Based Activities on Agitation, Affect, and Engagement in Nursing Home Residents with Dementia. International Psychogeriatrics, 25, 565-575. https://doi.org/10.1017/S1041610212002128

[31] Treusch, Y., Maji, T., Page, J., Gutzmann, H., Heinz, A. and Rapp, M.A. (2015) Apathy in Nursing Home Residents with Dementia: Results from Acluster-Randomized Controlled Trial. European Psychiatry, 30, 251-257. https://doi.org/10.1016/j.eurpsy.2014.02.004

[32] Chen, H.M., Tsai, L.J., Chao, S.Y. and Clark, M.J. (2016) Study on the Effects of Individualized Learning Therapy on Cognitive Function and Behavioral and Psychological Symptoms of Dementia in the Institutionalized Older Adults. The Journal of Nursing Research, 24, 300-310.

[33] Hoffman, K., Sobol, N.A., Fredriksen, K.S., Beyer, N., Vogel, A., Vestergaard, K., Braendgaard, H., Gottrup, H., Lolk, A., Wermuth, L., Jacobsen, L.P., Gergelyffy, H., Hogh, P., Bjerregaard, E., Andersen, B.B., Siersma, V., Johannsen, P., Cotman, C.W., Waldemar, G. and Hasselbalch, S.G. (2016) Moderate-to-High Intensity Physical Exercise in Patients with Alzheimer's Disease: A Randomized Controlled Trial. Journal of Alzheimer's Disease, 50, 443-453. https://doi.org/10.3233/JAD-150817

[34] Boström, G., Conradsson, M., Hörnsten, C., Rosendahl, E., Lindelöf, N., Holmberg, H., Nordström, P., Gustafson, Y. and Littbrand, H. (2016) Effects of a High-Intensity Functional Exercise Program on Depressive Symptoms among People with Dementia in Residental Care: A Randomized Controlled Trial. International Journal of Geriatric Psychiatry, 31, 868-878. https://doi.org/10.1002/gps.4401

[35] Chen, K.-M., Kuo, C.-C., Chang, Y.-H., Huang, H.-T. and Cheng, Y.-Y. (2017) Resistance Band Exercises Reduce Depression and Behavioral Problems of Wheelchair-Bound Older Adults with Dementia: A Cluster-Randomized Controlled Trial. Journal of the American Geriatrics Society, 65, 356-363. https://doi.org/10.1111/jgs.14526

[36] Lowery, D., Cerga-Pashoja, A., Iliffe, S., Thuné-Boyle, I., Griffin, M., Lee, J., Bailey, A., Bhattacharya, R. and Warner, J. (2014) The Effect of Exercise on Behavioural and Psychological Symptoms of Dementia: The EVIDEM-E Randomised Controlled Clinical Trial. International Journal of Geriatric Psychiatry, 29, 819-827. https://doi.org/10.1002/gps.4062

[37] McCabe, M.P., Bird, M., Davison, T.E., Mellor, D., MacPherson, S., Hallford, D. and Seedy, M. (2015) An RCT to Evaluate the Utility of a Clinical Protocol for Staff in the Management of Behavioral and Psychological Symptoms of Dementia in Residential Aged-Care Settings. Aging \& Mental Health, 19, 799-807. https://doi.org/10.1080/13607863.2014.967659

[38] Pieper, M.J.C., Francke, A.L., van der Steen, J.T., Scherder, E.J.A., Twisk, J.W.R., Kovach, C.R. and Achterberg, W.P. (2016) Effects of a Stepwise Multidisciplinary Intervention for Challenging Behavior in Advanced Dementia: A Cluser Randomized Controlled Trial. The American Geriatrics Society, 64, 261-269. https://doi.org/10.1111/jgs.13868

[39] Figueiro, M.G., Plitnick, B.A., Lok, A., Jones, G.E., Higgins, P., Hornick, T.R. and Rea, M.S. (2014) Tailored Lighting Interventions Improves Measures of Sleep, Depression and Agitation in Persons with Alzheimer's Disease and Related Dementia Living in Long-Term Care Facilities. Clinical Interventions in Aging, 9, 1527-1537. 
https://doi.org/10.2147/CIA.S68557

[40] Hickman, S.E., Barrick, A.L., Williams, C.S., Zimmerman, S., Connell, B.S., Preisser, J.S., Mitchell, C.M. and Sloane, P.D. (2007) The Effect of Ambient Bright Light Therapy on Depressive Symptoms in Persons with Dementia. Journal of American Geriatrics Society, 55, 1817-1824. https://doi.org/10.1111/j.1532-5415.2007.01428.x

[41] Yoshiyama, K., Arita, H. and Suzuki, J. (2015) The Effect of Aroma Hand Massage Therapy for People with Dementia. The Journal of Alternative and Complementary Medicine, 21, 759-765. https://doi.org/10.1089/acm.2015.0158

[42] Van Haitsma, K.S., Curyto, K., Abbott, K.M., Towsley, G.L., Spector, A. and Kleban, M. (2015) A Randomized Controlled Trial for an Individualized Positive Psychosocial Intervention for the Affective and Behavioral Symptoms of Dementia in Nursing Home Residents. Journal of Gerontology, 70, 35-45.

https://doi.org/10.1093/geronb/gbt102

[43] Thuné-Boyle, I.C., Iliffe, S., Cerga-Pashoja, A., Lowery, D. and Warner, J. (2011) The Effect of Exercise on Behavioral and Psychological Symptoms of Dementia: Towards a Research Agenda. International Psychogeriatrics, 24, 1046-1057. https://doi.org/10.1017/S1041610211002365

[44] Swedish Society of Nursing (2017) Strategy for Improving the Quality of Nursing. Swedish Society of Nursing, Stockholm.

https://www.swenurse.se/globalassets/01-svensk-sjukskoterskeforening/engelska/str ategy_for_improving_the_quality_of_nursing.pdf

[45] Novy, C. (2018) Life Stories and Their Performance in Dementia Care. The Arts in Psychotherapy, 57, 95-101. https://doi.org/10.1016/j.aip.2017.12.003

[46] Kitwood, T. (1997) Dementia Reconsidered: The Person Comes First. Open University Press, Buckingham.

[47] Kolanowski, A., Fick, D., Frazer, C. and Penrod, J. (2010) It's about Time: Use of Nonpharmacological Interventions in the Nursing Home. Journal of Nursing Scholarship, 42, 214-222. https://doi.org/10.1111/j.1547-5069.2010.01338.x

[48] Burack, O.R., Weiner, A.S. and Reinhardt, J.P. (2012) The Impact of Culture on Elder's Behavioral Symptoms: A Longitudinal Study. Journal of the American Medical Directors Association, 13, 522-528. https://doi.org/10.1016/j.jamda.2012.02.006 